Proc. of the X Int. Conf. - Ion Implantation and other Applications of Ions and Electrons, Kazimierz Dolny 2014

\title{
Depth Profile Analysis of Phosphorus Implanted SiC Structures
}

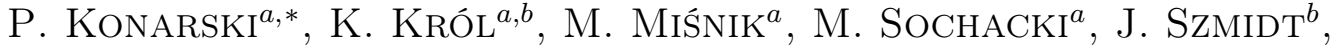 \\ M. TUREK ${ }^{c}$ AND J. ŻUK ${ }^{c}$ \\ ${ }^{a}$ Institute of Tele- and Radio Technology, Ratuszowa 11, 03-450 Warsaw, Poland \\ ${ }^{b}$ Institute of Micro- and Optoelectronics, Warsaw University of Technology, Koszykowa 75, 00-662 Warsaw, Poland \\ ${ }^{c}$ Institute of Physics, Maria Skłodowska-Curie University, Lublin, Poland
}

\begin{abstract}
Secondary ion mass spectrometry depth profile analyses were performed on two sets of $4 \mathrm{H}-\mathrm{SiC}(0001)$ substrate samples implanted with phosphorus. Both sets were processed under the same conditions. We implanted the samples with $100 \mathrm{keV}\left(10^{11}-10^{14} \mathrm{~cm}^{-2}\right)$ phosphorus ions through the thin chemical vapor deposition deposited silicon dioxide stopping mask in order to obtain an ultra-shallow implantation profile. After phosphorus implantation, secondary ion mass spectrometry depth profile analysis was performed on the first set of samples and the second set was subjected to thermal oxidation procedure at $1200{ }^{\circ} \mathrm{C}$ in order to create a dielectric layer. The aim of the oxidation process was formation of the silicon dioxide layer enriched with phosphorus: the element, which is considered to be suitable for trap density reduction. Ion implantation parameters as well as oxidation and chemical etching procedures were examined for the proper incorporation of phosphorus into the subsurface structure of the silicon oxide. Secondary ion mass spectrometry depth profile analysis was performed with Physical Electronics 06-350E sputter ion gun and QMA-410 Balzers quadrupole mass analyser. The analytical parameters such as: $1.7 \mathrm{keV} \mathrm{Ar}$ ion beam digitally scanned over $3 \times 3 \mathrm{~mm}^{2}$ area and ion erosion rate of $1.4 \mathrm{~nm} / \mathrm{min}$ and sampling rate of $0.3 \mathrm{~nm}$, were suitable for samples oxidized after ion implantation.
\end{abstract}

DOI: 10.12693/APhysPolA.128.864

PACS: 68.49.Sf, 68.55.Ln, 82.80.Ms, 85.40.Ry

\section{Introduction}

Secondary ion mass spectrometry (SIMS) is treated as one of the best techniques of elemental analysis of various materials due to its high sensitivity and high depth resolution. The SIMS technique is able to provide a semiquantitative analysis of bulk materials, however, it usually requires standards for quantitative analysis. Due to strong matrix effect, it is impossible to use relative sensitivity factors (RSFs) described for a given material during the analysis of another material [1]. Thus the quantitative depth profile analysis of heterostructures is highly difficult. This paper is devoted to the analysis of $\mathrm{SiO}_{2} / \mathrm{SiC}$ structure after shallow implantation with phosphorus ions.

The SIMS technique characterized the redistribution of common electrical dopants during the previous thermal oxidation of such structures [2]. It was shown that boron and aluminium depleted from the $\mathrm{SiC}$, while nitrogen and phosphorus were found to pile up in the $\mathrm{SiC}$, with the former being much more pronounced. The behaviour of phosphorus in the oxide remained unclear in this study due to the low concentration of the dopant.

The analysed in the present study structure works as a gate dielectric in silicon carbide $(\mathrm{SiC}) \mathrm{MOS}$ structure and is currently studied extensively due to its possible application in electronics. The phosphorus incorporation

*corresponding author; e-mail: piotr.konarski@itr.org.pl in this structure is considered to be the most suitable for trap density reduction [3].

\section{Experimental}

SIMS analyses were performed on two sets of $4 \mathrm{H}-$ $\mathrm{SiC}(0001)$ substrate samples implanted with phosphorus. Both sets were processed under the same conditions. For implantation we used $100 \mathrm{keV}$ phosphorus ions with the doses in the range $\left(10^{11}-10^{14} \mathrm{~cm}^{-2}\right)$. Implantation was performed through thin chemical vapour deposited silicon dioxide stopping mask in order to obtain an ultrashallow implantation profile. After phosphorus implantation, the SIMS depth profile analysis was performed on the first set of samples and the second set of implanted samples was subjected to thermal oxidation procedure at $1200^{\circ} \mathrm{C}$. This procedure was performed in order to create a dielectric layer. The oxidation process allowed to form a silicon dioxide layer enriched with phosphorus: the element, which is considered to be suitable for trap density reduction [3]. As a result of the oxidation process for samples $\# 1, \# 2, \# 3$, and $\# 4$ several values of oxide layer thickness: $42,62,41$, and $27 \mathrm{~nm}$, respectively, were obtained.

The SIMS depth profile analyses were made on the SAJW-05 analyser with the use of $500 \mathrm{nA}, 1.72 \mathrm{keV}$ $\mathrm{Ar}^{+}$primary ion beam at $45^{\circ}$ incidence angle. The analyser [4] is equipped with Physical Electronics 06-350E ion gun and QMA-410 Balzers quadrupole mass analyser with $16 \mathrm{~mm}$ diameter rods. Positive and negative secondary ion mass spectra were registered prior to the depth profile analyses. For analysis of the main components of the structure i.e. carbon, oxygen, silicon and 
phosphorus, a set of $m / z$ values: $12,16,28$, and 31 was chosen. Additionally $m / z=14$, a value representing nitrogen or double ionised silicon ions, was registered. In order to limit ion beam mixing effects there was used the ultra-low energy primary ion beam, which was digitally scanned, over a relatively large area $(3 \mathrm{~mm} \times 3 \mathrm{~mm})$. The secondary ions registered during the analysis are emitted from the $10 \%$ of the central area of that scanned. Using such primary beam parameters allows for high indepth resolution, however, it reduces also sensitivity with respect to the typical beam parameters such as $3 \mathrm{keV}$ and $1 \mu \mathrm{A}$.

\section{Results}

Raw data of depth profile SIMS analyses of the first set of ion implanted samples show relatively low phosphorus ion currents (Fig. 1). The $\mathrm{SiC}$ substrate, which was not implanted with phosphorus serves as a reference sample (ref.). Since absolute secondary ion currents do not represent atomic concentrations, we present ion current ratios (Fig. 2a). It can be observed that a not implanted reference sample shows also the presence of $m / z=31$ ions. These ions can originate from the molecular species such as ${ }^{30} \mathrm{SiH}^{+}$ions. To analyze results shown in Fig. 2a, the $I(31) / I\left(\mathrm{Si}^{+}\right)$and $I(31) / I\left(\mathrm{C}^{+}\right)$ratios for the reference sample were subtracted from the same ratios for first series samples $\# 1, \# 2$, and $\# 3$ - see Fig. 2b.

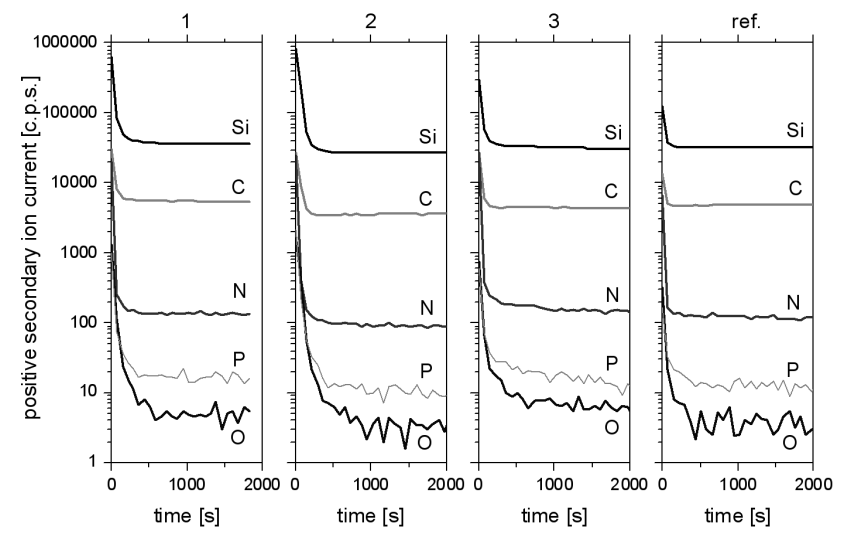

Fig. 1. SIMS depth profile analysis of positive secondary ions: $\mathrm{C}^{+}, \mathrm{N}^{+}, \mathrm{O}^{+}, \mathrm{Si}^{+}$and $\mathrm{P}^{+}$. Sputtering time $2000 \mathrm{~s}$ corresponds to a depth of $46 \mathrm{~nm}$ (sputtering rate is $1.4 \mathrm{~nm} / \mathrm{min}$ ). First set of samples: \#1, \#2 and $\# 3$ were phosphorus implanted with fluence $10^{11}$, $10^{12}$, and $10^{13} \mathrm{~cm}^{-2}$, respectively. Reference sample was not implanted.

Despite the fact that the measured phosphorus ion signals are weak, the results show that the phosphorus concentration distributions are different for the three samples.

The results of positive and negative SIMS analyses of the second set of samples, i.e. phosphorus implanted samples followed by the oxidation procedure, are shown in Fig. 3. After the oxidation procedure the structures show much differing phosphorus distributions. Different
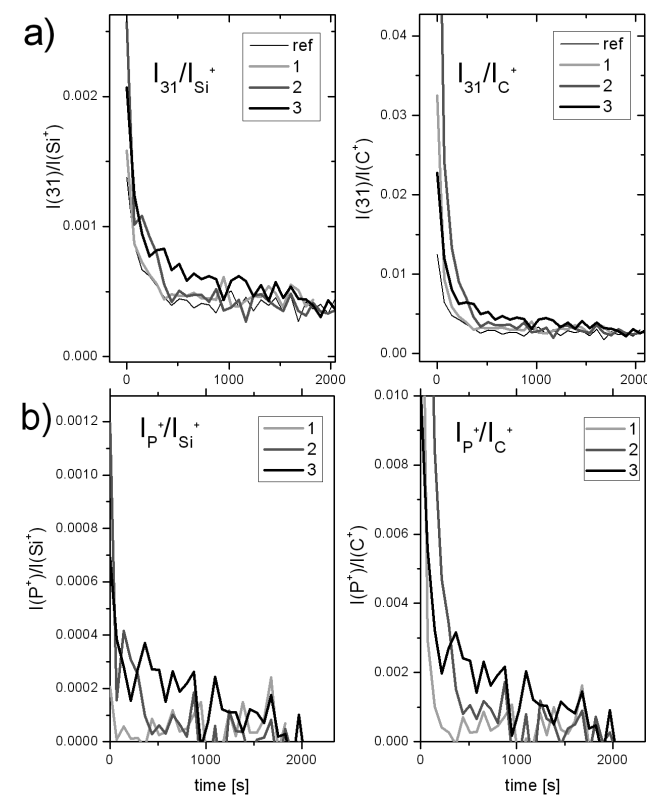

Fig. 2. SIMS depth profile analysis of phosphorus implanted samples (first series). The ion current ratios, $I(31) / I\left(\mathrm{Si}^{+}\right)$and $I(31) / I\left(\mathrm{C}^{+}\right)$, are plotted against the sputtering time: a) - without subtraction of values for the reference sample, b) - after subtraction of values for the reference sample.

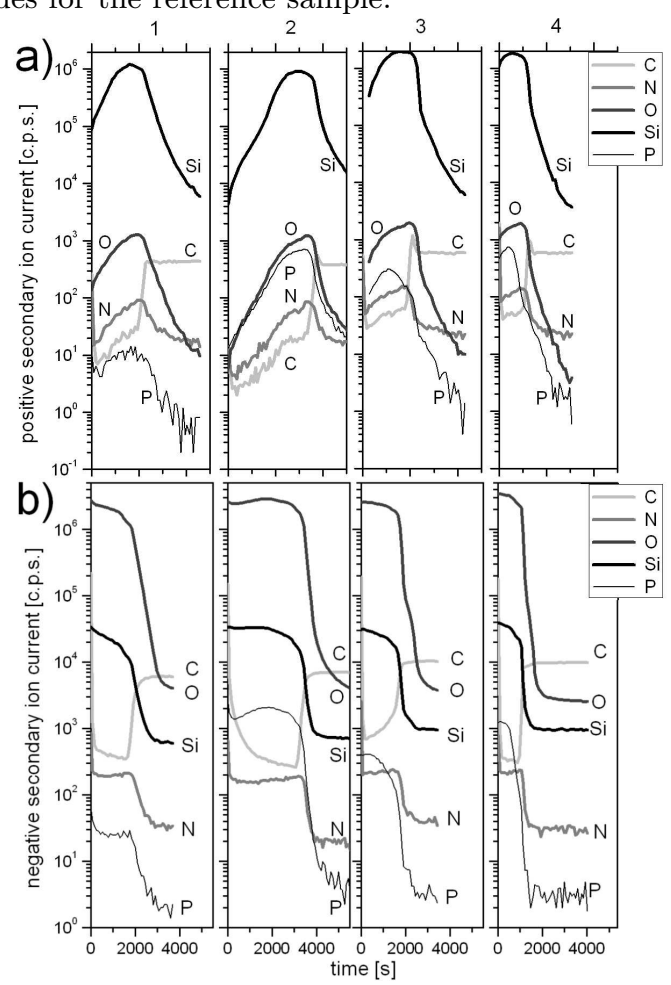

Fig. 3. SIMS depth profile analysis of second series samples $\# 1, \# 2, \# 3$, and $\# 4$, implanted with phosphorus fluence $10^{11}, 10^{12}, 10^{13}$ and $10^{14} \mathrm{~cm}^{-2}$, respectively, and oxidised in $1200^{\circ} \mathrm{C}$ after implantation. a) - positive secondary ion currents: $\mathrm{m} / z=12\left(\mathrm{C}^{+}\right)$, $14\left(\mathrm{~N}^{+}\right), 16\left(\mathrm{O}^{+}\right), 28\left(\mathrm{Si}^{+}\right), 31\left(\mathrm{P}^{+}\right)$and $\left.\mathrm{b}\right)-$ negative secondary ion currents: $m / z=12\left(\mathrm{C}^{-}\right), 14\left(\mathrm{~N}^{-}\right)$, $16\left(\mathrm{O}^{-}\right), 28\left(\mathrm{Si}^{-}\right), 31\left(\mathrm{P}^{-}\right)$are plotted versus sputtering time. 

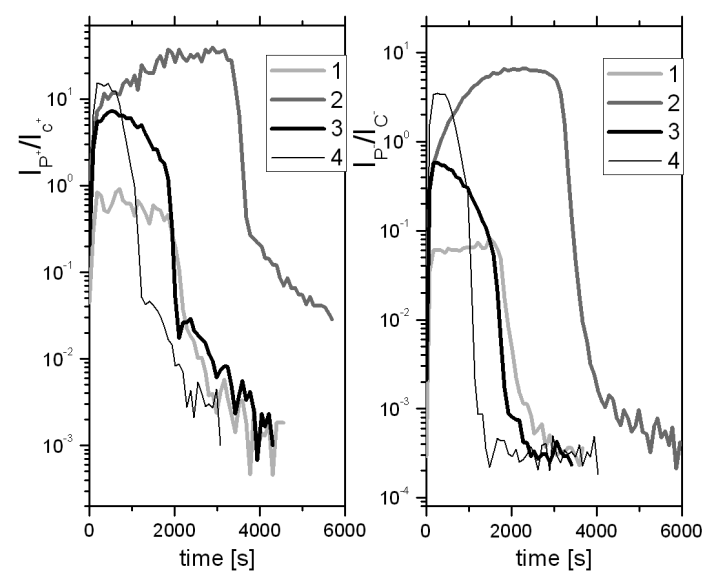

Fig. 4. SIMS depth profile analysis of positive (left panel) and negative (right panel) secondary ions for 2 -nd series samples $\# 1, \# 2, \# 3$, and $\# 4$. $I(\mathrm{P}) / I(\mathrm{C})$ ion current ratios are plotted versus sputtering time for phosphorus-implanted samples followed by thermal oxidation.

thicknesses and different levels of phosphorus secondary ion currents can be clearly seen. The $I(P) / I(C)$ ion current ratios of positive and negative ion currents of the oxidised samples are shown in Fig. 4 versus the sputtering time. As follows from Fig. 4, sputtering times of the consecutive sample oxide layers are closely related to the measured thickness values: $42,62,41$, and $27 \mathrm{~nm}$. One can also see that the $I(P) / I(C)$ ratio values in the vicinity of $\mathrm{SiO}_{2} / \mathrm{SiC}$ interface are different for the set of samples. Much sharper distributions of phosphorus at the interfaces are in samples " 1 " and "2", while much diffused distributions are in samples " 3 " and " 4 ".

\section{Conclusions}

In order to estimate atomic distributions in the analysed sets of samples, there are shown secondary ion ratios, which can possibly characterise implanted phosphorus distribution. The presented results show that the SIMS analytical parameters, for the analysis with the use of $\mathrm{Ar}^{+}$ion beam, are suitable for the analysis of shallow phosphorus implants in $\mathrm{SiC}$ if the implanted substrate is covered by the $\mathrm{SiO}_{2}$ layer. Characterisation of the implanted structures not covered by $\mathrm{SiO}_{2}$ (i.e. the first set of samples) is difficult, due to low efficiency of phosphorus secondary ion formation. In order to enhance this ionisation, the attempts were made to perform the SIMS analysis of the first set of samples using the analytical technique of oxygen flooding, however, no gain was observed. The fact that phosphorus ion emission is completely different in the two cases: during the analysis of $\mathrm{SiO}_{2}$ covered $\mathrm{SiC}$ substrate and during the analysis of $\mathrm{SiC}$ substrate with oxygen flooding, can be interpreted that the $\mathrm{SiO}_{2}$ coverage possibly influences some kind of phosphorus bonding like for example in $\mathrm{P}_{4} \mathrm{O}_{10}$ formation. Such chemical reaction could be beneath the $\mathrm{SiO}_{2}$ coverage, during its formation at $1200^{\circ} \mathrm{C}$, and perhaps could not be possible on the $\mathrm{Ar}^{+}$bombarded surface in the case of oxygen flooding in the SIMS analytical chamber up to $10^{-6}$ mbar of partial pressure. Another possible explanation of the differences in phosphorus SIMS signals in the two sample sets is that in the case of the first set, ion removal of atomic layers during the SIMS analysis enables phosphorus to evaporate into vacuum.

\section{Acknowledgments}

This paper was partially supported by the National Science Centre (Poland) grant No. UMO$2012 / 05 / \mathrm{N} / \mathrm{ST} 7 / 02035$. This work was co-financed by European Social Fund.

\section{References}

[1] P.C. Zalm, Vacuum 45, 753 (1994).

[2] J.W. Palmour, R.F. Davis, H.S. Kong, S.F. Corcoran, D.P. Griffis, J. Electrochem. Soc. 136, 502 (1989).

[3] D. Okamoto, H. Yano, T. Hatayama, T. Fuyuki, Appl. Phys. Lett. 96, 203508 (2010).

[4] P. Konarski, A. Mierzejewska, Appl. Surf. Sci. 203204, 354 (2003). 